\title{
La technique du vote en amphithéâtre
}

\author{
Jean-Luc CRACOWSKI ${ }^{1}$
}

\section{Définition}

Technique pédagogique utilisable en amphithéâtre pour faciliter l'activité des étudiants.

\section{Objectif}

Favoriser la participation active des étudiants à un cours magistral, sans limite de nombre (plusieurs centaines d'étudiants).

\section{Intérêt}

Cette technique est associée à la conduite du cours magistral.

Elle incite les étudiants à être attentifs à l'intégralité du cours et permet d'augmenter le temps d'attention.

Elle augmente l'interactivité étudiant-enseignant et l'interactivité entre les étudiants.

Par son aspect ludique et participatif, elle rompt avec la relation classique enseignant-enseigné.

Il s'agit d'une des rares techniques favorisant l'activité des étudiants au sein de très grands groupes, sans rendre nécessaire le fractionnement de l'auditoire en groupes plus restreints.

\section{Préparation}

- Préparation de la séquence pédagogique par l'enseignant

- Définir les objectifs pédagogiques principaux et secondaires.

- Expliciter le plan du cours, construire son support didactique (diapositives, transparents,...).

- Identifier les champs d'interrogation des étudiants selon trois types d'objectifs :

Réflexion/application : les étudiants doivent réfléchir à partir des éléments fournis précédemment pour élaborer la réponse adéquate.

Connaissance : la question concerne des éléments impor- tants du cours que les étudiants vont devoir restituer.

Sondage : la question vise à informer l'enseignant de l'opinion de l'assistance sur un point particulier, non évoqué précédemment. Ceci permet de tester certaines connaissances antérieures des étudiants ou de stimuler leur attention en rendant le cours participatif.

- Rédiger les " quiz ". Cette étape est essentielle. Les quiz doivent être centrés sur les objectifs principaux. A ce titre, les quiz de synthèse, à la fin d'un chapitre, sont particulièrement utiles. Penser à faire varier le style (quiz de réflexion, de connaissance et de sondage). Le rythme idéal est d'un quiz toutes les 10/15 minutes, soit 3 à 6 quiz par heure de cours. Les quiz correspondent forcément à des questions à choix simple, ce qui est la principale limite du système.

- Tester les quiz : modifier les quiz trop faciles ou trop difficiles. Le caractère flou d'un énoncé n'est pas forcément un problème s'il force l'étudiant à examiner différentes alternatives en lien avec une question judicieusement choisie.

\section{Réalisation}

\section{- Mise en place en amphithéâtre}

La première mise en place nécessite d'être effectuée soigneusement pour favoriser l'adhésion du public cible : il est recommandé de débuter avec un premier quiz facile, éventuellement humorisitique.

\section{- Techniques}

Deux techniques peuvent être utilisées :

\section{- Les cartons de couleur}

Il s'agit de la technique la plus simple.

Déroulement de la séquence:

Elle consiste à distribuer en début séance quatre cartons de couleur vive, qui serviront de bulletin de vote. Le principe est simple, mais doit être très clairement énoncé, avec quatre temps : 1) formulation de l'énoncé du quiz ; 2) réflexion personnelle et silencieuse des étudiants ; 3) sélection par chaque étudiant, sans le montrer,

1. Faculté de médecine - Département de pharmacologie - INSERM ERI 17 - Université Joseph Fourier (UJF) 38706 Grenoble - France. Mailto:Jean-Luc.Cracowski@ujf-grenoble.fr

Travail effectué dans le cadre du Diplôme d'université de pédagogie médicale (UJF - Grenoble) 
du carton correspondant à son choix ; 4) vote simultané de tous les étudiants en levant haut les cartons.

Le respect de la séquence est primordial pour éviter un phénomène de contamination des votes (étudiant n'ayant pas de réponse et regardant ses collègues!).

Avantages et inconvénients:

Nécessité d'une très bonne gestion du groupe par l'enseignant pour éviter la cohue dans l'amphithéâtre, excellente interactivité car chaque étudiant voit la réponse de son voisin et peut donc en discuter ; évaluation semi-quantitative des réponses par l'enseignant facilité par le recours aux couleurs, qui permet de discerner d'un coup d'œil les réponses prédominantes.

\section{- Le vote informatique}

Chaque étudiant dispose d'un boîtier électronique relié à un logiciel permettant de donner le pourcentage de réponse à chaque item.

Avantages et inconvénients : aspect très " jeu télévisé », qui peut plaire ou agacer un public francophone, simplicité de la procédure car les étudiants ne voient pas les votes des autres, ce qui peut cependant être un inconvénient.

Déroulement du vote :

Quelle que soit la modalité utilisée, une fois le vote effectué, deux possibilités :

- La réponse juste est très majoritaire. Dans ce cas, la notion est supposée comprise et l'enseignant continue le cours.

- Il existe une hétérogénéité des votes. L'enseignant peut inviter les étudiants à discuter pendant trois minutes avec leurs voisins, de manière à ce qu'ils comparent et argumentent brièvement leurs réponses. Un deuxième vote est ensuite organisé ; il est fréquent que la bonne réponse devienne majoritaire, sans intervention de l'enseignant, ce qui atteste d'une appropriation des notions exposées en amphithéâtre.

\section{Debriefing}

\section{Debriefing personnel}

À l'issue du cours, pour tous les nouveaux quiz, faire l'analyse de la façon dont se sont déroulés les votes (quiz trop faciles, trop difficiles ou ambigus) ; les modifier immédiatement, le cas échéant.

Debriefing collectif

A l'issue d'un ou deux cours, vérifier l'adhésion des étudiants en effectuant un vote de type sondage.

\section{Des conseils pratiques}

- Les étudiants francophones ne sont pas plus réticents à ce type d'enseignement que les étudiants anglosaxons, lancez-vous!

- Lors de cours successifs, il est très important de débuter par un quiz de synthèse sur le cours précédent.

- Un quiz prend trois à cinq minutes ; penser à raccourcir le cours d'autant.

- Un quiz peut carrément remplacer une séquence d'exposé déclaratif.

- A la fin de chaque quiz, expliciter le bien fondé ou le caractère erroné des différentes réponses alternatives.

\section{Coût et ergonomie}

Les cartons de couleur sont très économiques. Il convient cependant de préparer le matériel : couper des cartons de quatre couleurs (format A4 divisé en 4). Le procédé impose à l'enseignant de venir avec un stock large de cartons pour que tous les étudiants en disposent. Pour des cours successifs, laisser les cartons sur place et les récupérer lors du dernier cours

Le vote informatique nécessite un système électronique de vote relativement coûteux mais facilite la tâche de l'enseignant lorsque le système marche.

\section{Variante}

Cette technique peut être utilisée après un travail à domicile, qui peut consister par exemple en une activité de résolution d'exercice avec l'aide d'un corpus de référence. Dans ce cas, les étudiants travaillent au préalable, guidés par des objectifs pédagogiques. La séance consiste à travailler par quiz à partir des apprentissages effectués par les étudiants. Il est possible de discuter en groupe les notions mal acquises.

\section{Conclusion}

La technique du vote en amphithéâtre peut être utilisée pour l'ensemble de l'enseignement magistral. Elle nécessite une préparation soigneuse de la part de l'enseignant pour être réussie et atteindre ses objectifs. Elle permet de soutenir l'attention, même pour des sujets difficiles, et de rendre les étudiants actifs. 